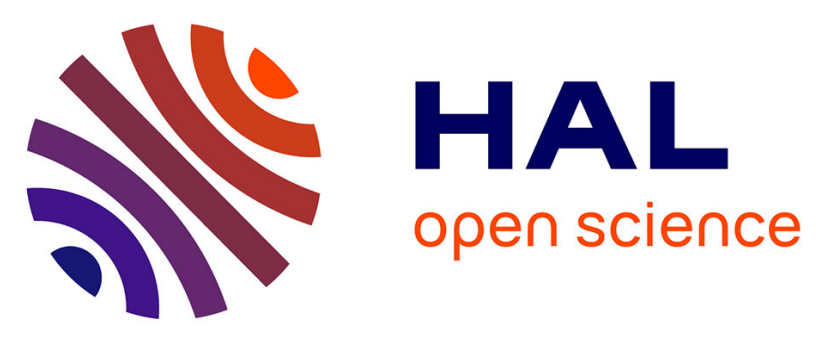

\title{
Melting-Induced Evolution of Morphology, Entanglement Density, and Ultradrawability of Solution-Crystallized Ultrahigh-Molecular-Weight Polyethylene
}

Fotis Christakopoulos, Egor Bersenev, Souren Grigorian, André Brem, Dimitri A Ivanov, Theo A Tervoort, Victor Litvinov

\section{To cite this version:}

Fotis Christakopoulos, Egor Bersenev, Souren Grigorian, André Brem, Dimitri A Ivanov, et al.. Melting-Induced Evolution of Morphology, Entanglement Density, and Ultradrawability of SolutionCrystallized Ultrahigh-Molecular-Weight Polyethylene. Macromolecules, 2021, 54 (12), pp.5683-5693. 10.1021/acs.macromol.1c00667 . hal-03453462

\author{
HAL Id: hal-03453462 \\ https://hal.science/hal-03453462
}

Submitted on 28 Nov 2021

HAL is a multi-disciplinary open access archive for the deposit and dissemination of scientific research documents, whether they are published or not. The documents may come from teaching and research institutions in France or abroad, or from public or private research centers.
L'archive ouverte pluridisciplinaire HAL, est destinée au dépôt et à la diffusion de documents scientifiques de niveau recherche, publiés ou non, émanant des établissements d'enseignement et de recherche français ou étrangers, des laboratoires publics ou privés. 
Melting-Induced Evolution of Morphology, Entanglement Density and UltraDrawability of Solution-Crystallized Ultra-High Molecular Weight Polyethylene.

Fotis Christakopoulos, ${ }^{1}$ Egor Bersenev, ${ }^{2,3}$ Souren Grigorian, ${ }^{4}$ André Brem, ${ }^{1}$ Dimitri A. Ivanov, ${ }^{2,3,5}$ Theo A. Tervoort, ${ }^{1}$ Victor Litvinov, ${ }^{6 *}$

${ }^{1}$ ETH Zürich, Vladimir-Prelog-Weg 5, 8093 Zürich, Switzerland

${ }^{2}$ Lomonosov Moscow State University, 119991 Moscow, Russian Federation

${ }^{3}$ Institute of Problems of Chemical Physics, Russian Academy of Sciences, Chernogolovka, Moscow Region 142432, Russian Federation

${ }^{4}$ Institute of Physics, University of Siegen, D-57068 Siegen, Germany

${ }^{5}$ Institut de Sciences des Matériaux de Mulhouse-IS2M, CNRS UMR 7361, F-68057 Mulhouse, France

${ }^{6}$ V.Lit.Consult, Gozewijnstraat 4, 6191WV Beek, The Netherlands

ABSTRACT: The melting-induced change in density of physical network junctions, which are formed by chain entanglements and network junctions due to anchoring of chain segments to crystals, is studied by ${ }^{1} \mathrm{H}$ NMR $T_{2}$ relaxometry for solution- and melt-crystallized ultra-high molecular weight polyethylene (UHMWPE) - sc-UH and mc-UH, respectively. The NMR results are complemented by real-time synchrotron WAXS and SAXS analyses to extract the sizes of the crystalline lamellae and inter-crystalline domains. Below the melting temperature, the network of physical junctions is denser in the amorphous phase of mc-UH than the one in scUH owing to lower entanglement density and smaller number of physical junctions from polymer crystals in sc-UH. However, the difference in the total density of physical junctions between mc-UH and sc-UH films decreases with decreasing crystallinity during melting. At the end of the melting trajectory, at vanishing crystallinity, the volume-average entanglement density, as characterized by the NMR method, is approximately the same in sc- and mc-UH. This indicates that the entanglement density in sc-UH films increases during melting owing to fast buildup of local chain entanglements. These entanglements are formed by segments of the same chain, neighboring chains, or the both due to a displacement of chain fragments upon lamellar thickening and due to the so-called "chain explosion" that occurs locally in the amorphous domains. The increase in the entanglement density in sc-UH is additionally confirmed by solid-state drawability of sc-UH films that were annealed in the melting region but below the end of melting. The maximum draw ratio decreases and the drawing stress increases with increasing annealing temperature.

\section{INTRODUCTION}

An intrinsic property of long chain molecules are chain entanglements which originate from the uncrossability of neighboring chains surrounding a given chain. The degree of entanglement is characterized by the entanglement density, which is one of the fundamental characteristics of polymer melts determining their viscoelastic properties. In solid polymers, the entanglement density influences fracture behavior, the strength of interfacial adhesion in polymer composites, the strain-hardening modulus during plastic deformation and the resistance to creep failure by 
slow crack growth in the case of semi-crystalline polymers. ${ }^{1,2,3,4,5}$ Entanglements significantly influence the nucleation rate during crystallization, ${ }^{6}$ the size of crystalline and amorphous domains, and long-range order in adjacent re-entry sequence of chains in the crystal lamellae, whereas this order can hardly be modified by the experimentally accessible crystallization conditions. ${ }^{7}$ Entanglement density is also a key parameter when determining the solid-state drawability at elevated temperatures of "soft" semicrystalline polymers that exhibit main chain dynamics in the crystalline phase. ${ }^{8}$ It is widely accepted for these systems that drawability increases with decreasing entanglement density. ${ }^{9,10}$ The maximum draw ratio, $\lambda_{\max }$, which is measured in the solid state at elevated temperatures for melt-crystallized high-density polyethylene (HDPE) and ultrahigh-molecular-weight polyethylene (UHMWPE), does not exceed $6-9,{ }^{11,12}$ whereas disentangled films and single-crystal mats of HDPE and UHMWPE can be drawn to draw ratios of $250-370 .^{12,13}$

Polymers with a decreased entanglement density can be realized in different ways. Crystallization of polymers from the equilibrium melt causes a heterogeneous distribution of chain entanglements, ${ }^{14,15,16}$ and can lead to a decrease in their density. ${ }^{17,18}$ Crystallization of polymers from a sheared melt can also result in a decrease of the entanglement density. ${ }^{19}$ The entanglement density and morphology in semicrystalline polymers are affected by the polymer crystallization and crystallization conditions for solution-crystallized materials ${ }^{7,10,20,21}$ and by the type of catalyst and polymerization conditions for nascent powders. ${ }^{22,23}$ Low-entangled, extended-chain crystals are obtained by crystallization of HDPE at high pressure. ${ }^{24}$ Differences in the entanglement density are reflected in the phase composition, in the chain motion in polyethylene (PE) crystals, the $\alpha$-relaxation process in crystals, in long-range chain diffusion between crystalline and amorphous domains, and in the type of the crystal-amorphous interface, namely, switchboard-like for melt-crystallized PE and the adjacent-reentry-like morphology with short loops on the lamellae surface for low-entangled PEs. The entanglement density reaches its equilibrium state upon melting of semicrystalline polymers. There are two opposing views on the rate of the re-entangling after melting of low-entangled polymers, i.e., slow- and fast recovery to the equilibrium melt state. Terms like "melt memory" 25 , and "chain explosion"26 or "explosion upon melting" 27 are often used to describe these phenomena, respectively.

Conflicting statements being made on the melt memory effect in polyethylene's. The existence of a low-entangled melt state has been shown by a crystallization study of molten high-pressure-crystallized HDPE with medium molecular weight $\left(M_{\mathrm{w}}=55000 \mathrm{~kg} / \mathrm{mol}, M_{\mathrm{n}} / M_{\mathrm{w}}\right.$ $=4.80) .{ }^{28}$ Despite the short reptation time of approximately $0.1 \mathrm{sec},{ }^{26}$ a low entanglement density persisted in the HDPE melt for 20-30 min as was concluded from a fast spherulite growth rate. Slow heating $\left(0.2^{\circ} \mathrm{C} / \mathrm{min}\right)$ of low-entangled nascent UHMWPE powders provided a long-lived-heterogeneous low-entangled melt state as was shown by different methods. ${ }^{29,30,31}$ Contrary to these results, close to instantaneous recovery to the equilibrium melt state was shown by a neutron scattering study on the melting of solution-grown PE crystals. ${ }^{26}$ Chainfolded crystals "explode" upon melting and the fold energy of tight polymer coils is released in a spring-wise manner, resulting in an ultra-fast interpenetration of chains, often referred to as "chain explosion". The chains virtually immediately adopt a random-coil conformation due to the entropy-driven process. Fast equilibration of entanglement density upon melting was shown by the instantaneous loss of drawability in solution-cast low-density polyethylene (LDPE) and UHMWPE, ${ }^{11}$ and by the fast auto-adhesion of solution-crystallized nascent UHMWPE films. ${ }^{32,33}$ The same conclusion was recently made in a study of sintering of UHMWPE powder. ${ }^{34}$ The study suggested that the entropy-driven, fast re-entangling process is due to segmental dynamics 
rather than reptation of entire chains. Thus, the reptation model is perhaps not strictly applicable in this case since molecules must move "sideways" as well as along their length to achieve the random coil conformation, occupy a larger volume and share this volume with many other molecules, as was also suggested earlier by de Gennes. ${ }^{27}$

Recently, a methodology was developed for the estimation of the volume-average entanglement density in semicrystalline polyolefins by studying HDPEs in melting temperature range with ${ }^{1} \mathrm{H}$ NMR $T_{2}$ relaxometry. ${ }^{35}$ Melting-induced changes in the phase composition and chain motion in the crystalline and the amorphous phases were followed by the real-time NMR experiments. It was shown that the network of physical junctions in the amorphous phase of semicrystalline polymers is composed of junctions originating from chain anchoring to crystals and chain entanglements. To estimate the entanglement density in the amorphous phase, $T_{2}$ relaxation experiments were performed at temperatures close to and gradually approaching melting where the density of crystal-induced physical junctions vanishes. The aforementioned study detected a lower entanglement density at the end of melting for high-pressure-crystallized HDPE compared to its melt-crystallized counterpart. ${ }^{35}$ Application of this new method is of interest in studying the phase composition, molecular motions and the density of physical network junctions in the melting temperature range of solution-crystallized UHMWPE. Given that the ultra-drawing at elevated temperatures of such materials results into high strength/high modulus fibers, knowledge of melting-induced changes would provide a better understanding of the eff ect of temperature on the ultra-drawing process. It is, therefore, the objective of this study is to evaluate these meltinginduced changes in physical structures and the structure of network that is composed of crystalinduced physical junctions and chain entanglements in low-entangled, solution-crystallized and isotropic melt-crystallized UHMWPE. First, the morphology and the physical structures of solutionand melt-crystallized UHMWPE below the melting onset temperature are characterized. Then, changes in morphology, phase composition and total density of physical junctions in the amorphous phase are recorded upon melting. Last, the role of the physical network junctions in the stress-strain behavior of solution-crystallized UHMWPE films is examined. ${ }^{1} \mathrm{H}$ NMR $T_{2}$ relaxometry is used (i) to determine changes in the phase composition and molecular mobility upon melting, and (ii) to study the effect of melting on the total density of physical network junctions, which are formed by chain entanglements and physical junctions originating from chain anchoring to crystals. ${ }^{35}$ Synchrotron wide- and small-angle X-ray scattering (WAXS and SAXS) experiments are used to follow changes in crystallinity, crystal thickness and thickness of interlamellar amorphous layers in the melting temperature range. The experiments reflect reorganization of the crystal structure which can affect the structure of the network of physical junctions. The solid-state drawability experiments are utilized to evaluate the role of the entanglement density in the stress-strain behavior of solution-crystallized UHMWPE films. Since the structural reorganization on heating is a time- and temperature-dependent phenomenon, ${ }^{36,37,38}$ it was decided to perform structural, thermal and NMR experiments at similar heating ramp conditions in order to obtain comparable results. Finally, the role of the entanglement density in the solid-state drawability of solution-crystallized UHMWPE films is discussed.

\section{EXPERIMENTAL SECTION}

2.1. Samples and Their Preparation. Commercial UHMWPE, GUR4120 ${ }^{\circledR}$, was supplied by Ticona. The average molecular weight of the polymer was calculated from the intrinsic viscosity [ $\eta$ ] using the Margolies' equation and is equals to $4700 \mathrm{~kg} / \mathrm{mol}$ according to supplier 
info. For the preparation of solution-crystallized films, nascent UHMWPE powder was dissolved in decalin at $160{ }^{\circ} \mathrm{C}$, stirred for $1 \mathrm{~h}$ under $\mathrm{N}_{2}$ flow and fast crystallized upon cooling of the solution with a rate of $>20{ }^{\circ} \mathrm{C} / \mathrm{min}$. To prevent degradation of UHMWPE, 1 weight part of Irganox/Irgafos stabilizer per 100 weight parts of the polymer was added to decaline before heating. After crystallization, the samples were dried at room temperature. Three different concentrations of the UHMWPE were used for preparing the solutions that ranged from approximately 1 to $10 \mathrm{wt} \%$. The exact concentrations were calculated from the weight of the solutions and the weight of UHMWPE films after solvent evaporation and there equals to 1.31, 3.67 and $10.6 \mathrm{wt} \%$. The following designation of samples is used: sc-UH-1.3; sc-UH-3.7; scUH-10.6 and mc-UH where the numbers provide the concentration of UHMWPE in the solutions, and sc-UH and mc-UH stand for solution- and melt-crystallized UHMWPE, respectively. Additionally, a film crystallized from a $20 \mathrm{wt} \%$ solution was prepared in the same way. This film was used for dynamic mechanical thermal analysis (DMTA) and for solid-state drawability experiments.

Thermal Analysis and Thermal Characteristics of UHMWPE Films. Thermal analysis was conducted at heating and cooling rates of $10{ }^{\circ} \mathrm{C} / \mathrm{min}$ on a Discovery 2500 differential scanning calorimeter (TA Instruments, USA). The sample mass was approximately $2 \mathrm{mg}$. Indium standard was used for temperature and heat flow calibrations. DSC thermograms were recorded under a nitrogen flow of $20 \mathrm{ml} / \mathrm{min}$ and are shown in Part 1.1 of the Supporting Information. The crystallinity was calculated from the enthalpy of melting, $\Delta \mathrm{h}$, of the first DSC heating run, using

the relation $x=\frac{\Delta h}{\Delta h_{f}}$ where $\Delta \mathrm{h}_{\mathrm{f}}$ is the heat of fusion of polyethylene crystals equals to $293 \mathrm{~J} / \mathrm{g}$. ${ }^{39}$ Thermal characteristics of the films are shown in Table 1. To resemble the heating protocol during the X-ray analyses, DSC measurements at a rate of $1{ }^{\circ} \mathrm{C} / \mathrm{min}$ were performed with a NETZSCH DSC214 Polyma instrument. Samples were cut from the films and placed in $30 \mu 1 \mathrm{Al}$ pans with pierced lids. Then, the pans were heated from 25 to $180{ }^{\circ} \mathrm{C}$. The thermograms for scUH-1.3 and mc-UH are shown in Part 1.1 of the Supporting Information.

Table 1. Thermal Characteristics of UHMWPE Films Evaluated for the First Heating at a Rate of $10{ }^{\circ} \mathrm{C} / \mathrm{min}$

\begin{tabular}{|l|c|c|c|c|c|}
\hline \multicolumn{1}{|c|}{ sample } & $\begin{array}{c}\text { solution } \\
\text { concentration, } \\
\mathrm{wt} \%\end{array}$ & $\begin{array}{c}\text { heat of } \\
\text { fusion, } \\
\mathrm{J} / \mathrm{g}\end{array}$ & $\begin{array}{c}\text { crystallinity, } \\
\mathrm{wt} \%\end{array}$ & $\begin{array}{c}\text { melting onset } \\
\text { temperature, }{ }^{\circ} \mathrm{C}\end{array}$ & $\begin{array}{c}\text { peak melting } \\
\text { temperature, }\end{array}$ \\
\hline sc-UH-1.3 & 1.3 & 212 & 72 & 125 & 132.7 \\
\hline sc-UH-3.7 & 3.7 & 205 & 69 & 125 & 132.4 \\
\hline sc-UH-10.6 & 10.6 & 197 & 67 & 124 & 132.2 \\
\hline mc-UH & - & 124 & 42 & 121 & 134.0 \\
\hline
\end{tabular}

2.2. Solid-state drawability. Dumbbell-shaped bars were cut from the UHMWPE films according to ISO 527-2 type 5B. The bars were drawn at $120{ }^{\circ} \mathrm{C}$ with an Instron 5864 static mechanical tester at an initial strain rate of $0.1 \mathrm{~s}^{1}$. The mean maximum draw ratio $\left(\lambda_{\max }\right)$ was determined from at least five measurements for each film.

2.3. Dynamic Mechanical Thermal Experiments. DMTA experiments were conducted on an ARES G2 rheometer (TA Instruments, USA) at $110^{\circ} \mathrm{C}$. The temperature was controlled in an oven with a forced $\mathrm{N}_{2}$ convection using a Pt100 temperature sensor which was in a close 
proximity to the samples. The heating rate was $2{ }^{\circ} \mathrm{C} / \mathrm{min}$. The measurement was conducted at a frequency of $1 \mathrm{~Hz}$ and a strain amplitude of $0.5 \%$. The samples were always under tension during the measurements by applying a constant force of $0.1 \mathrm{~N}$ which caused only minor extension. Changes in the position of the clamps due to the thermal expansion of the setup were calibrated in advance with an iron strip of known thermal expansion coefficient. Drawing of the partially molten samples was performed immediately after the DMTA measurement was finished without taking films out of the clamps with the initial strain rate of $0.05 \mathrm{~s}^{-1}$.

2.4. X-Ray Synchrotron Experiments. Temperature-resolved SAXS and WAXS experiments were performed at the P03 beamline of PETRA III storage ring (Hamburg, Germany) and at the ID02 beamline of ESRF (Grenoble, France). The energy of the incident beam was set in both cases to $12.4 \mathrm{keV}$ and 2D scattering patterns were recorded using hybrid photon-counting detectors. The recorded 2D data were centred, calibrated, regrouped, and reduced to $1 \mathrm{D}$ profiles using the SAXS utilities platform described elsewhere, ${ }^{40}$ and custom designed Python routines based on PyFAI library. ${ }^{41}$ In the experiments, samples were heated at 1 ${ }^{\circ} \mathrm{C} / \mathrm{min}$ rate to the final melting region with a Linkam heating stage. Solution-crystallized films were placed in a custom-built transverse sample holder so that the film plane was parallel to the beam direction. The synchrotron experiments were complimented by laboratory-based SAXS machine Xenocs Xeuss equipped with GeniX 3D sealed tube, evacuated sample chamber that was connected with a flight tube and Pilatus 300k detector. The microstructural parameters of the semicrystalline morphology were analysed by calculating $1 \mathrm{D}$ correlation functions, ${ }^{42}$ and $1 \mathrm{D}$ chord distribution functions ${ }^{43,44,45}$ similar to that in our previous publication. ${ }^{35}$

2.5. Time-Domain ${ }^{1}$ H NMR Experiments. ${ }^{1} \mathrm{H}$ NMR transverse magnetization relaxation ( $T_{2}$ relaxation) experiments were performed for static samples on a Bruker Minispec MQ20 relaxometer equipped with $10 \mathrm{~mm}$ temperature probe head. Detailed information on the equipment and experiments performed was provided previously. ${ }^{35}$ Small pieces of films were randomly packed in an NMR tube. The NMR tube with samples at room temperature was placed into the NMR probe-head which was stabilized at the required temperature. Five minutes were required for temperature stabilization within the sample volume. ${ }^{35,46}$ This time was taken as zero in all real-time NMR $T_{2}$ relaxation experiments. To determine relative change in the density of physical network junctions in the amorphous phase at melting temperatures, $T_{2}$ decay was recorded as a function of time at several temperatures. A new sample was used for the experiments at each temperature. Each experiment took fewer than 2 min.

2.6. Phase Composition of Samples by ${ }^{1} \mathrm{H}$ NMR $T_{2}$ Relaxometry. A three-phase model was used for determining the phase composition and molecular mobility in different phases as characterized by $T_{2}$ relaxation time. In this model, a crystal-amorphous interface separates the crystalline and the amorphous domains. A linear combination of an Abragamian, a Gaussian and exponential functions provided the best description of the $T_{2}$ decay for solution-crystallized UHMWPE films at $70{ }^{\circ} \mathrm{C}$ (equation 1)

$A(t)=A(0)^{\mathrm{cr}} \exp \left[-\left(t / 2 T_{2}^{\mathrm{cr}}\right)^{2}\right] \cdot[\sin (\alpha t) / \alpha t]+A(0)^{\mathrm{in}} \exp \left[-\left(t / T_{2}^{\mathrm{in}}\right)^{2}\right]+A(0)^{\mathrm{am}} \exp \left[-\left(t / T_{2}^{\mathrm{am}}\right)\right]$

The three relaxation components were assigned to the crystalline phase - $\left(T_{2}^{\text {cr }}\right.$ relaxation time); the crystal-amorphous interface - $\left(T_{2}^{\text {in }}\right.$ relaxation time); and the amorphous phase - $\left(T_{2}{ }^{\text {am }}\right.$ relaxation time). The relative amount of highly mobile chain end segments in the UHMWPE 
was very small. ${ }^{35}$ Therefore, the relaxation of the amorphous phase was approximated by the single exponential function. The longer the $T_{2}{ }^{\text {index }}$ for the same phase, the larger the frequency of molecular motions, their amplitude, or both are. It should be noted that difference between the values of $T_{2}{ }^{\mathrm{cr}}, T_{2}{ }^{\mathrm{in}}$ and $T_{2}{ }^{\mathrm{am}}$ cannot be used as a measure of relative difference in molecular mobility in the different phases due to the difference in the shape factor of these relaxation components, i.e., Abragamian, Gaussian and exponential shapes, and difference in the origin of NMR relaxation processes for chain segments with different molecular mobility. The relative fraction of the relaxation components, $\left\{A(0)^{\text {index }} /\left[A(0)^{\mathrm{cr}}+A(0)^{\mathrm{in}}+A(0)^{\mathrm{am}}\right]\right\} \times 100 \%$, represents the relative amount of hydrogen atoms (as expressed in weight fractions) of the crystalline phase, the crystal-amorphous interface, and the amorphous phase, respectively. Value of $\alpha$ in the Abragamian function for the crystalline phase is provided in Table SI-1 in the Supporting Information. Since values of the $T_{2}$ relaxation parameters depend on the fitting function used, ${ }^{47,48}$ equation 1 was also used for the analysis of $T_{2}$ decay of all samples in entire temperature range studied.

2.7. Network Density in Rubbery Materials and Chain Entanglements in HDPE Melts by ${ }^{1}$ H NMR Relaxometry. NMR relaxometry is a well-established methodology for characterization of network structure in viscoelastic materials. The presence of network junctions, either chemical cross-links, physical junctions or both, increases the anisotropy of chain motions which causes an increase in the strength of interactions between nuclear spins. These internuclear interactions are quantified by different NMR experiments and are used for calculating the molecular weight of network chains. ${ }^{49,50,51}$ The $T_{2}$ relaxation method is a simple and robust way for such analysis providing quantitative data on molecular weight of network chains in polymers with chemical crosslinks, (quasi)permanent physical junctions, or both. ${ }^{52,53,54}$ In polymer melts, the fluctuation of entanglement nodes (in the discrete binary entanglements model) or the tube diameter (in the tube model) at the time scale of the NMR $T_{2}$ relaxation experiment $\left(\sim T_{2}\right.$ value) causes an overestimation of molecular weight between apparent chain entanglements, $M_{e}$. The effect is small for UHMWPE as was shown by a theoretical analysis of $T_{2}$ decay for a series of HDPE melts with a large range of molecular weight. ${ }^{55}$ The obtained $M_{\mathrm{e}}$ value of $1760 \mathrm{~g} / \mathrm{mol}^{55}$ exceeds a typical $M_{\mathrm{e}}$ value of $1250 \mathrm{~g} / \mathrm{mol}$ which is commonly accepted by rheologists. ${ }^{56}$ However, this value is in the $M_{\mathrm{e}}$ range reported previously for HDPEs. ${ }^{55}$ It is noted that different methods should not necessary provide the same $M_{\mathrm{e}}$ value due to several reasons discussed previously. ${ }^{55}$

2.8. Chain Entanglements in the Amorphous Phase of Melt-Crystallized HDPE in the Melting Temperature Range. A methodology for estimation the volume-average entanglement density in amorphous phase of HDPEs was recently developed and is used in the present study. ${ }^{35}$ The method is based on analysis of the dependence of $1 / T_{2}{ }^{\text {am }}$ relaxation rate of the amorphous phase in the melting temperature range against the remaining crystallinity. As an example, the dependence is shown for melt-crystallized polymer - (mc-HDPE) in Figure $1 .^{35}$ At temperatures well above $T_{\mathrm{g}}$, the NMR $T_{2}$ relaxation time of the amorphous phase is determined not only by chain entanglements but also by junctions which originate from polymer crystals providing a dense network in the amorphous phase. ${ }^{35}$ The contribution from polymer crystals to the total network density in mc-HDPE is the major component at temperatures below melting. Upon melting of mc-HDPE, the number of crystal-induced physical junctions decreases and chain entanglements provide a larger contribution to the total network density. The dependence of $1 / T_{2}{ }^{\mathrm{am}}$ against crystallinity for mc-HDPE reaches an asymptotic value, $1 / T_{2}{ }^{\mathrm{am}}(0)$, at a crystallinity below $10 \mathrm{wt} \%{ }^{35}$ The value of $1 / T_{2}{ }^{\mathrm{am}}(0)$ is used for estimation of the weight- 
average-molecular-weight of chain segments between apparent chain entanglements, $M_{\mathrm{e}}$, as described previously. ${ }^{35}$ It was shown that re-entangling of mc-HDPE in melting temperature range hardly occurs for high-molecular-weight polymers due to restriction on long-spatial scale segmental mobility from remaining crystals. ${ }^{35}$ Value of $1 / 2 M_{\mathrm{e}}$ corresponds to the volumeaverage entanglement density. This NMR experiment cannot detect heterogeneous distribution of chain entanglements in the amorphous phase. The entanglement density in the amorphous phase in the semicrystalline state is higher than its value in the melt since most of the entanglements cannot be disentangled during rapid crystallization of HDPE and preexisting entanglements in the melt are pushed to the inter-lamellar layers by the growing crystals. ${ }^{14,16,57}$

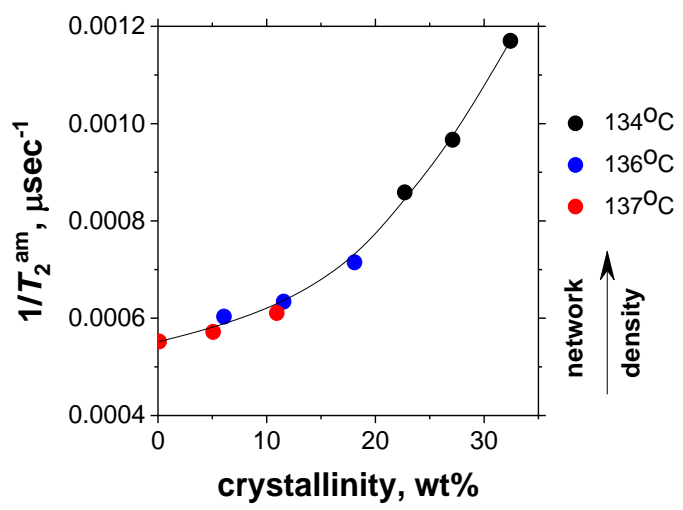

Figure 1. Dependence of $1 / T_{2}{ }^{\text {am }}$ against crystallinity for melt-crystallized HDPE. ${ }^{35}$ The polymer has $M_{\mathrm{n}}=95 \mathrm{~kg} / \mathrm{mol}$ and $M_{\mathrm{w}}=260 \mathrm{~kg} / \mathrm{mol}$. Crystallinity decreases with time at the same temperature due to partial melting. ${ }^{35}$

\section{RESULTS AND DISCUSSION}

3.1. Morphology and Physical Structures in Solution- and Melt-Crystallized UHMWPE Films Below Melting Temperature. The lamellar thickness and long period of the periodic lamellar structure are determined by SAXS and the obtained values are summarized in Table 2. Lamellar thickness in all sc-UH films is approximately two times smaller than that in mc-UH due to reasons discussed previously. ${ }^{58}$ The thickness of interlamellar amorphous layers $l_{\mathrm{a}}$, in mc-UH is approximately $10 \mathrm{~nm}$. Such large value is entirely due to the effect of chain entanglements. Pre-existing entanglements in the UHMWPE melt are pushed by the growing crystals to interlamellar layers and restrict further crystal growth resulting in nearly two times lower crystallinity and much thicker amorphous layers in mc-UH (Table 2). The thickness of interlamellar non-crystalline layers in sc-UH is only $2 \mathrm{~nm}$ and approximately equals to the size of chain folds separating adjacent lamellae. The amount of the crystal-amorphous interface, which is determined by the NMR method, is nearly the same for all sc-UH films and equals $13 \pm$ $1 \mathrm{wt} \%$ (Table SI-1 in the Supporting Information). Despite the higher crystallinity and the smaller lamellar thickness in sc-UH, the fraction of the interface relative to crystallinity $\left(\% T_{2}{ }^{\text {in }} / \% T_{2}{ }^{\mathrm{cr}}\right)$ is approximately 1.5 times smaller than its value for mc-UH as shown by ${ }^{1} \mathrm{H}$ NMR $T_{2}$ relaxation experiment (Table SI-1 in the Supporting Information). This is indicative of tight chain folds on the lamellar surface having a large fraction of adjacent chain re-entering into the same lamellae. ${ }^{59}$ Chain mobility in the crystal-amorphous interface of sc-UH is larger than that in mc-UH. This can be due to faster chain-diffusion in and out of crystals that has been shown by high-field ${ }^{13} \mathrm{C}$ NMR experiments. ${ }^{59}$ 
The crystallinity of the samples was independently evaluated through WAXS, SAXS and NMR and the values obtained are presented in Table 2. The values measured for each sample differ depending on the measurement method due to several reasons as discussed previously. ${ }^{60}$ However, the same trend of increasing crystallinity with decreasing initial polymer concentration is observed for all experimental approaches. The crystallinity of the sc-UH samples is found to be nearly two times higher compared to the mc-UH, as was expected due to the lower entanglement density in sc-UH. In addition, the $T_{2}{ }^{\mathrm{cr}}$ value for sc-UH is lower than mc-UH (Table SI-1 in the Supporting Information), thus suggesting a more perfect crystal packing in sc-UH, resulting in smaller average internuclear distances and, consequently, stronger proton-proton interactions.

Table 2. Crystallinity of UHMWPE Films as Determined by NMR, WAXS and SAXS. Lamellar Thickness $\left(L_{c}\right)$, Thickness of Interlamellar Amorphous Layers $\left(L_{\mathrm{a}}\right)$ and Long Period $\left(L_{\mathbf{p}}\right)$ as Determined by SAXS Experiments for Solution- and Melt-Crystallized UHMWPE Films at Room Temperature.

\begin{tabular}{|c|c|c|c|c|c|c|}
\hline \multirow[t]{2}{*}{ sample } & \multicolumn{3}{|c|}{ crystallinity } & \multirow[t]{2}{*}{$L_{\mathrm{c}},{ }^{(\mathrm{c})} \mathrm{nm}$} & \multirow[t]{2}{*}{$L_{\mathrm{a}},{ }^{(\mathrm{c})} \mathrm{nm}$} & \multirow[t]{2}{*}{$L_{\mathrm{p}},{ }^{(\mathrm{c})} \mathrm{nm}$} \\
\hline & $\begin{array}{c}\text { WAXS } \\
\text { crystallinity } \\
\text { index, wt } \%\end{array}$ & $\begin{array}{c}\text { SAXS } \\
\text { linear } \\
\text { crystallinity, } \\
\%\end{array}$ & $\begin{array}{c}\text { NMR } \\
\text { crystallinity, } \\
\text { wt } \%\end{array}$ & & & \\
\hline sc-UH-1.3 & 86 & 81 & 83.5 & 8.9 & 2.1 & 11.0 \\
\hline sc-UH-3.7 & 83 & 73 & 82.5 & 8.5 & 3.1 & 11.6 \\
\hline sc-UH-10.6 & 72 & n.a. ${ }^{\text {(a) }}$ & 81.0 & n.a. & n.a & 14.9 \\
\hline mc-UH & 45 & 61 & 46.5 & 16.2 & 10.4 & 26.6 \\
\hline
\end{tabular}

(a) not analyzed

(b) NMR crystallinity was determined at $70{ }^{\circ} \mathrm{C}$ and obtained values are shown Part 1.2 in the Supporting Information

(c) parameters extracted from the Interface Distribution Functions.

3.2. Evolution of the Size of Crystalline and Amorphous Domains Upon Melting of Solution- and Melt-Crystallized UHMWPE Films. The changes in dimensions of crystalline and amorphous domains in melting temperature range can provide information on the order of translational displacements of chain segments that can lead to re-entanglement ${ }^{35}$. The evolution of the microstructural parameters for the two extreme cases of the studied samples, i.e. samples sc-UH-1.3 and mc-UH, is assessed by analysis of synchrotron SAXS and WAXS data recorded during a heating ramp of $1.0^{\circ} \mathrm{C} / \mathrm{min}$. The results are shown in Figure 2. In panel A of Figure 2, it can be seen that the SAXS curve of the sc-UH exhibits three orders of the main interference peak, whereas for the mc-UH only one broad peak is clearly visible. This difference reflects the order in stacking of lamellar crystals which is much higher for the sc-UH-1.3 film. This is also illustrated by the Interface Distribution Functions (IDF) shown in panel B of Figure 2. One observes the classical shape of the IDF for the sc-UH-1.3, displaying separated distributions of both the crystalline and amorphous regions. By contrast, the mc-UH displays a much broader distribution of peaks which are almost merged together. Upon melting, the crystals of the sc-UH1.3 undergo a dramatic reorganization whereby their thickness starts to significantly increase below the WAXS crystallinity index of ca. 0.7 . Clearly, the heating rate of $1.0^{\circ} \mathrm{C} / \mathrm{min}$ is slow 
enough to allow for lamellar thickening to occur, resulting to almost doubling of the initial crystal thickness towards the end of the melting region. In contrast, the crystals in mc-UH, being much thicker below the melting temperature, display smaller variation in thickness upon melting. This limited thickening could be partially due to the high entanglement density in the amorphous phase. A longer timescale would be required for the thickening process to proceed. The occurrence of lamellar thickening can be also concluded from the corresponding DSC traces that are shown in Figure SI-1.2 of the Supporting Information. For the slowest heating rate applied, the melting peak exhibits a high-temperature shoulder.

A
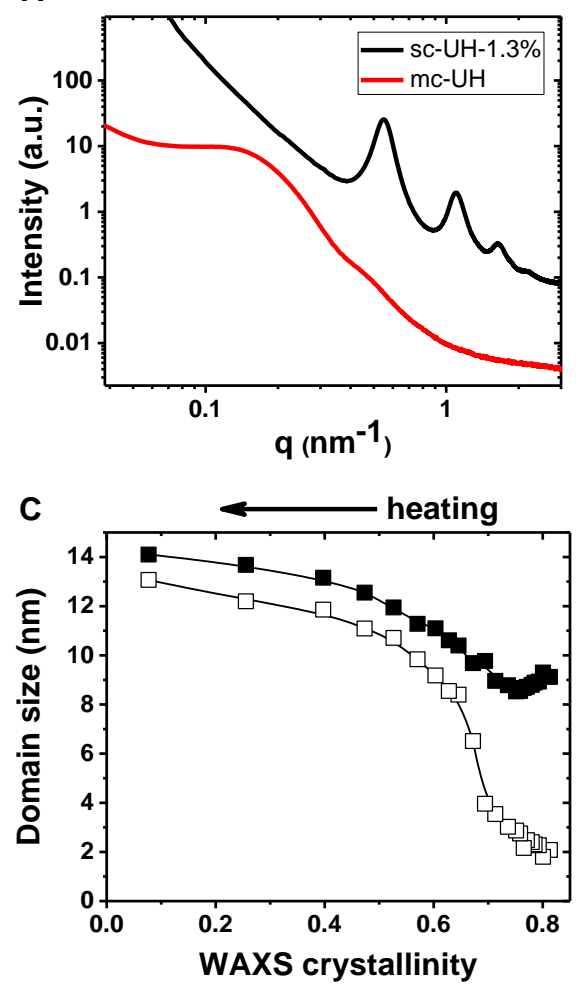

B
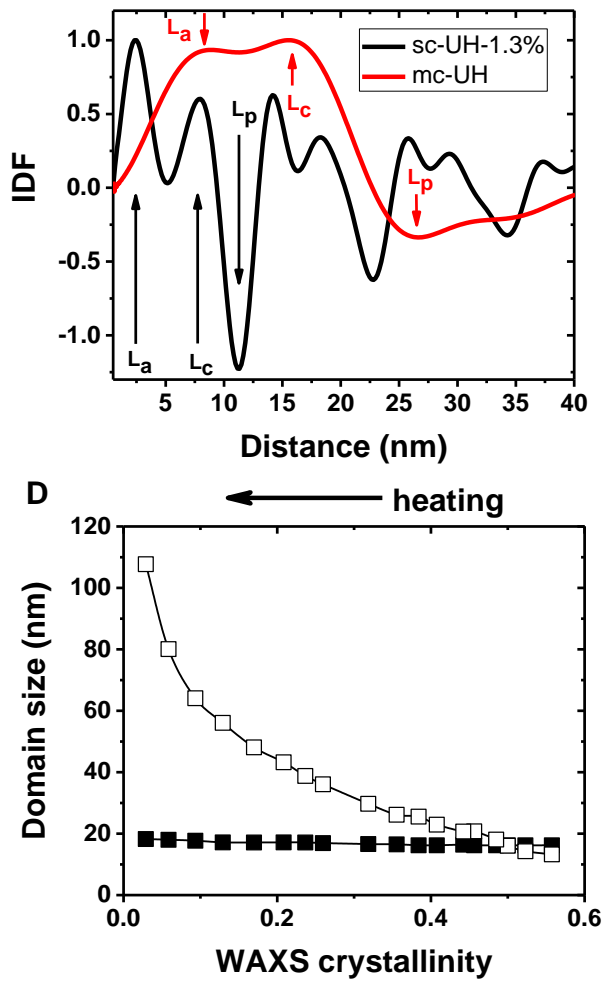

Figure 2. Room-temperature SAXS curves of sc-UH-1.3 and mc-UH (A); and the corresponding Interface Distribution Functions (B). Arrows indicate most probable thickness for corresponding domains. Domain sizes are calculated from the Interface Distribution Functions for sc-UH-1.3 (C), and mc-UH (D) films. Filled squares correspond to lamellar thickness and empty squares - to thickness of interlamellar amorphous layers. Line between symbols is a guide to the eye.

As far as the evolution of the amorphous regions upon heating is concerned, their characteristic size dramatically increases for both samples. The thickness of amorphous layers for the sc-UH-1.3 film progressively increases from ca. $2 \mathrm{~nm}$ to more than $13 \mathrm{~nm}$ at the later stage of melting (Figure 2C). Interestingly, the final value of the amorphous layer thickness for the solution-crystallized sample is roughly equal to the corresponding initial value for the meltcrystallized sample (Figure 2D). This suggests that the increasing amount of the amorphous phase in sc-UH-1.3 could be also accompanied by an increasing entanglement density. It is noteworthy that linear SAXS crystallinity becomes much higher than the crystallinity index extracted from WAXS at advanced stages of melting. This can be accounted for by the fact that 
the linear crystallinity represents the morphology within the ordered lamellar stacks, whereas the WAXS crystallinity index takes into account all the 3D structures comprising not only the stacks but also the growing molten regions surrounding them.

3.3. Change in the Total Density of Physical Junctions in the Amorphous Phase Upon Melting of Solution- and Melt-Crystallized UHMWPE Films. Melt Memory or "Chain Explosion"? The total density of physical junctions in the amorphous phase is composed of chain entanglements and crystal-induced physical junctions and is characterized by $1 / T_{2}{ }^{\text {am }} \cdot{ }^{35}$ The lower the $1 / T_{2}{ }^{a m}$, the smaller the network density is. Since the effect of $1 / T_{2}{ }^{a}$ depends on crystallinity, the difference in the network structure in mc-UH and sc-UH should be compared at the same crystallinity. The dependence of $1 / T_{2}$ am against crystallinity in melting temperature range is shown for mc- and sc-UH in Figures 1 and 3, respectively. The change in $1 / T_{2}{ }^{a m}$ with decreasing crystallinity is caused by four processes: ${ }^{35}$ (1) a decrease in the number of crystalinduced physical junctions leading to a decrease in $1 / T_{2}{ }^{\mathrm{am}} ;(2)$ if no re-entangling would occur, $1 / T_{2}{ }^{\text {am }}$ will decrease due to dilution of the local entanglement network in the amorphous phase since its volume increases at constant overall entanglement density; (3) a decrease in confinement of chain segments in the amorphous phase by adjacent crystal lamellae, the socalled the "corset effects", ${ }^{61}$ should cause a decrease in $1 / T_{2}{ }^{\mathrm{am}}$; and (4) an increase in entanglement density, if it occurs, will cause an increase in $1 / T_{2}{ }^{\mathrm{am}}$. The combination of these four processes determines the total change in $1 / T_{2}$ am upon melting.

The effects of crystals play a dominant role in the decrease of $1 / T_{2}{ }^{\text {am }}$ upon melting of mc$\mathrm{UH}$, because entanglement density provides a smaller contribution to the total density of the physical junctions. ${ }^{35}$ Therefore, the decrease in crystallinity causes a large decrease in $1 / T_{2}{ }^{\mathrm{am}}$ for mc-UH. Assuming that no re-entangling occurs upon melting, the volume-average entanglement density in the amorphous phase of mc-UH should additionally decrease due to the dilution of existing entanglements as a result of the increasing amount of amorphous phase. In addition, melting also causes a decrease in confinement of chain segments by adjacent crystal lamellae due to increasing thickness of interlamellar amorphous layers both in mc-UH and sc-UH (Figure 2,C-D). The larger the thickness of the amorphous layers, the smaller the confinement and its effect on $1 / T_{2}$ am .

At a crystallinity of $\sim 40 \%$, the $1 / T_{2}{ }^{\text {am }}$ for partially molten sc-UH films is significantly lower compared to mc-UH. This is due to a lower density of chain entanglements and a smaller amount of crystal-induced physical junctions in the amorphous phase of sc-UH, as was shown in Section 3.1. If the overall entanglement density would be constant during melting of sc-UH, it would be expected that $1 / T_{2}{ }^{a m}$ for sc-UH will gradually decrease upon decreasing crystallinity due to progressive dilution of the entanglements and the effects of decreasing crystal-induced physical junctions. In this case, $1 / T_{2}{ }^{a m}$ at vanishing crystallinity of sc-UH should reach a lower value than that for mc-UH. However, it can be seen in Figure 3 that the experimentally observed difference between $1 / T_{2}{ }^{\text {am }}$ for mc- and sc-UH decreases with decreasing crystallinity and approaches nearly the same value at the end of melting. This indicates the increase in the volume-average entanglement density in sc-UH, which becomes approximately the same in mc-UH and sc-UH films at crystallinity below $\sim 10 \%$. This observed increase in the entanglement density in sc-UH cannot be caused by chain reptation since residual crystals limit diffusional motion of entire UHMWPE chains and the time of the NMR experiments is at least two orders of magnitude smaller than the reptation time, $\tau_{\text {rep }}$, of UHMWPE chains. A neutron scattering study determined a reptation time of $\tau_{\text {rep }}=2.3$ hours for UHWMPE with $M_{\mathrm{w}}=4000 \mathrm{~kg} / \mathrm{mol}{ }^{26}$ For the UHMWPE used in the present study $\left(M_{\mathrm{w}}=4700 \mathrm{~kg} / \mathrm{mol}\right)$, a reptation time of approximately 4.1 hours is 
estimated through the following equation: $\tau_{\text {rep }}=3 \cdot 10^{-7}\left(M_{\mathrm{w}} / 1350\right)^{3}$. Therefore these results suggest that the volume-average entanglement density in the amorphous phase of sc-UH films increases with decreasing crystallinity during melting and approaches approximately the same value as previously determined for melt-crystallized UHMWPE. ${ }^{35}$

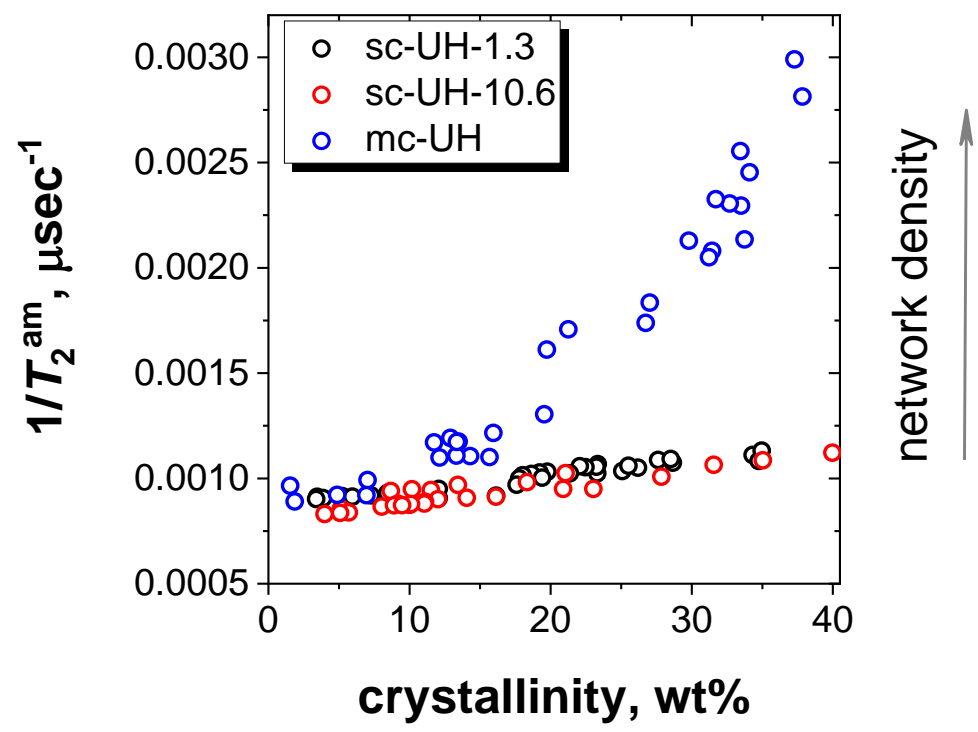

Figure 3. Dependence of $1 / T_{2}{ }^{\text {am }}$ against crystallinity which is determined as a function of time at different temperatures for solution-and melt-crystallized UHMWPE films. Only the data points, which are recorded during 2-4 min after the samples reached the required temperature, are included in the graph.

This increase in entanglement density during melting of sc-UH can be explained by the formation of local chain entanglements, by a localized "chain explosion", or the both. A twostep formation of entanglements upon melting of low-entangled-high-molecular weight polymers has been suggested previously. ${ }^{62}$ At a first step, local chain entanglements are formed by the same chain, by neighboring chains, or the both due to chain twists, formation of knots and loops. This type of entanglements can be easily formed within a short time without significant long-spatial-scale chain diffusion. At longer times, equilibration of entanglements, through the polymer volume, occurs in the molten state. This process requires reptation of all chains and is excluded for partially molten polymers since polyethylene crystals largely slow down chain diffusion. The increase in entanglement density can be also explained by the so-called "chain explosion" which occurs locally in amorphous domains involving only a fraction of chain coils.

In order to understand the molecular origin of the formation of local chain entanglements, melting-induced changes in morphology and chain dimensions should be considered. One of the possible mechanisms is lamellar thickening which causes local displacement of chains in amorphous domains between adjacent lamellae. The radius of gyration $R_{\mathrm{g}}$ of solutioncrystallized HDPEs is proportional to $M^{1 / 4}-M^{1 / 2}{ }^{63}$ The estimated $R_{\mathrm{g}}$ for the studied sc-UH films is in the range from 19 to $36 \mathrm{~nm}$. This range corresponds to approximately two- to three long periods of sc-UH films (Table 2). The typical lateral lamellar size of solution-crystallized HDPEs exceeds micrometers. ${ }^{64}$ Thus, each crystal lamella is built by many polymer chains that need to be locally displaced during the lamellar thickening process. The thickness of the 
amorphous layers $\left(L_{\mathrm{a}}\right)$ is two-three times smaller than the radius of gyration of sc-UH and $L_{\mathrm{a}}$ in sc-UH-1.3 increases by a few times during the initial stage of melting. Such a large change should lead to interweaving - local entangling of polymer chains. Reorganization of the chainfolding-pattern during lamellar thickening may also cause the formation of latent chain entanglements. ${ }^{65}$ The other mechanism, which can explain the rapid increase in entanglement density, is a localized "chain explosion" in the amorphous domains. A localized "chain explosion" can explain the large increase in the thickness of the amorphous layers which is already observed for sc-UH during the moderate crystallinity decrease at the initial stage of melting (Figure 2C). A neutron scattering study of melting of HDPE-single-crystal-mats has shown that a regular super-folded sheet structure in crystallites, which has a rather compact nonequilibrium conformation, adopts the random-coil-configuration in the melt within a very short time. "The molecule springs out, driven by the need to increase its entropy" upon melting. The localized "chain explosion" mechanism is in agreement with drawability and welding studies on solution-crystallized UHMWPE films which were molten and immediately quenched prior drawing and welding experiments. ${ }^{11,32,33}$ These studies have shown that viscoelastic properties in the solid state and in the melt are governed by segmental dynamics rather than by mobility of entire chains. Consequently, reptation of chains as a whole appears not to be a prerequisite for restoring equilibrium properties of low-entangled HDPE and UHMWPE.

3.4. The Effect of Entanglements on Drawability of Solution-Crystallized UHMWPE Films. In order to investigate whether the detected by NMR entanglement-formation during melting affects mechanical properties, the solid-state drawability of solution-crystallized UHMWPE films was studied.

3.4.1. Solid State Drawability of Solution-Crystallized UHMWPE Films Prior Annealing at Melting Temperatures. Entanglement density is a key parameter which determines the solid-state drawability of semicrystalline polymers. ${ }^{9,10,66}$ For UHMWPE, it generally assumed that the maximum drawability, $\lambda_{\max }$, which is measured at elevated temperatures but below the melting point, scales as the ratio of the fully stretched chain length to the random-walk end-to-end distance of a chain segment between entanglements, resulting in the following scaling rule: ${ }^{10}$

$$
\lambda_{\max }=\frac{N_{e} l_{b}}{\sqrt{C_{\infty} N_{e} l_{b}^{2}}}=\sqrt{\frac{N_{e}}{C_{\infty}}} \propto \sqrt{M_{e}}
$$

Here $N_{\mathrm{e}}$ is the number of monomer units between neighbouring entanglements along a chain, $l_{\mathrm{b}}$ is the bond length and $C_{\infty}$ is the characteristic ratio. Assuming that the molecular weight between entanglements in solution is inversely proportional to the polymer volume concentration, $\phi,{ }^{67}$ and that the crystallization process does not influences the entanglement density, it follows that the maximum drawability of UHMWPE-films crystallized from solution and after removal of the solvent, scales as

$$
\lambda_{\max } \propto \sqrt{M_{e}} \propto \frac{1}{\sqrt{\phi}}
$$

The maximum drawability values of all UHMWPE samples were determined at $120{ }^{\circ} \mathrm{C}$ and are plotted in a double-logarithmic plot against the polymer volume fraction of the solutions from which they were cast (Figure 4). The slope of -0.48 is in agreement with other studies. ${ }^{10}$ and with the scaling relation derived above. This confirms that the low entanglement density of the solutions is preserved during subsequent casting, crystallization and drying. Thus, the resulting solution-crystallized films that were used for the NMR and X-ray diffraction experiments are disentangled to various degrees. 


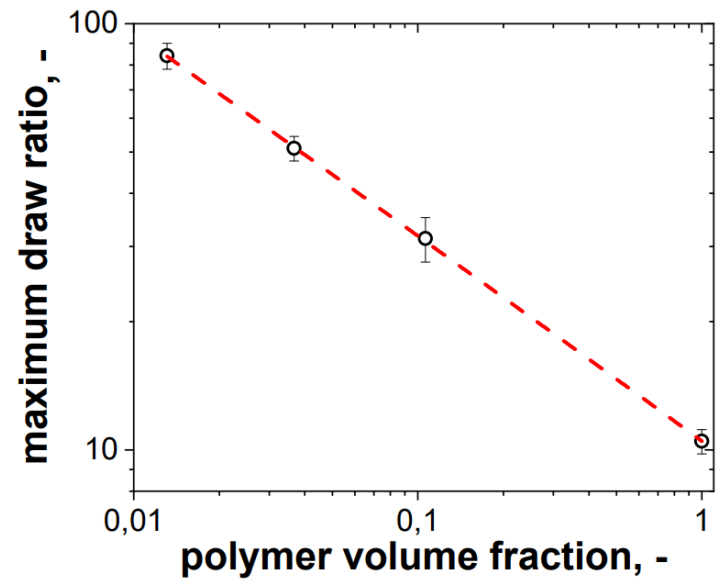

Figure 4. Maximum draw ratio of solution-crystallized UHMWPE films at $120{ }^{\circ} \mathrm{C}$ against polymer volume fraction. The red dotted line corresponds to a nonlinear model fit $f(\mathrm{x})=A \mathrm{x}^{n}$, with $A=10.6 \pm 0.8$ and $n=-0.48 \pm 0.02$.

\subsubsection{Solid-State Drawability of Solution-Crystallized UHMWPE Films After Partial}

Melting. The NMR experiments show an increase in the entanglement density upon approaching complete melting. In addition, it is widely accepted that an increased entanglement density would hinder ultra-drawability and should cause an increase in the associated stress needed for drawing. ${ }^{66}$ In order to justify or disprove the observed by NMR increase in entanglement density, the solid-state drawability is studied for partially molten sc-UH films.

The melting onset and the end of melting of the $20 \mathrm{wt} \%$ solution-crystallized UHMWPE film are observed at $\sim 128{ }^{\circ} \mathrm{C}$ and $136{ }^{\circ} \mathrm{C}$, respectively. The storage modulus of the film largely decreases upon heating to temperatures above the onset of melting (Figure 5). This is mainly caused by the decrease in crystallinity, hence supporting that the samples are indeed partially melting. After the required annealing temperature was reached, the film was immediately quenched under a strong nitrogen flow without removing the sample from the clamps. The partially molten fraction of UHMWPE crystallizes upon cooling. Then, the temperature of the annealed sample was stabilized at $110{ }^{\circ} \mathrm{C}$ and stretched in the solid-state. The drawability curves of stripes cut from the film which were heated to 128,130131 and $135{ }^{\circ} \mathrm{C}$ are presented in Figure 6. If the entanglement density would not change during partial melting followed by crystallization, the stress-strain curves should be identical for all samples. However, a large change in the stress-strain properties is observed after annealing at elevated temperatures above the melting onset but below the end of melting. Both the yield stress and the strain-hardening increase, whereas the maximum draw ratio drastically decreases with increasing annealing temperature. The increase in strain-hardening and decrease in the maximum draw ratio upon annealing both indicate an increase in the entanglement density. Thus, the results of the solidstate drawability experiment are in agreement with the rapid entanglement formation as shown by the NMR method. The observed partial loss of drawability after partial melting is also consistent with the complete loss of drawability after heating of solution-crystallized LDPE and UHMWPE into the melt for a short period of time. ${ }^{11}$ 


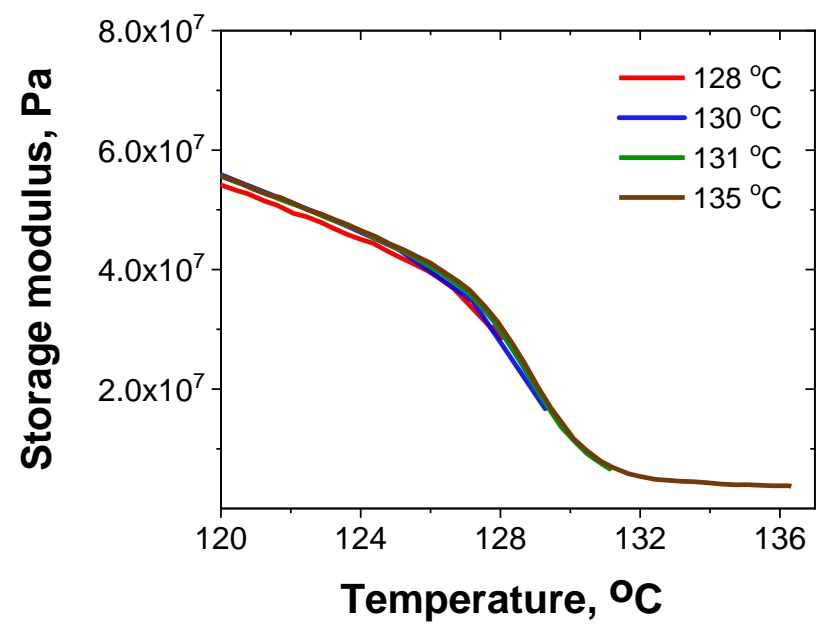

Figure 5. Storage modulus of stripes cut from a sc-UH film against temperature. The film was solution-casted from a $20 \mathrm{wt} \%$ UHMWPE solution. The storage modulus was recorded during film heating with the rate of $2{ }^{\circ} \mathrm{C} / \mathrm{min}$, frequency of $1 \mathrm{~Hz}$ and at a strain amplitude of $0.5 \%$. A new film was used for each measurement with different maximum heating temperatures.

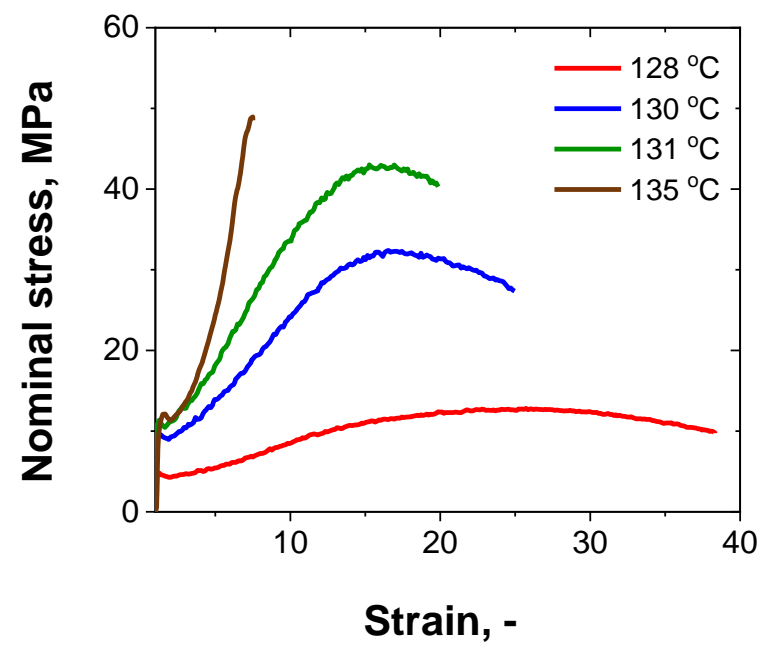

Figure 6. Solid-state drawability curves of stripes cut from a sc-UH film annealed at different temperatures. The film was solution-casted from a $20 \mathrm{wtl} \%$ UHMWPE solution. Drawing was performed at $110{ }^{\circ} \mathrm{C}$ with initial strain rate of $0.05 \mathrm{~s}^{-1}$. Melting onset is observed at $\approx 128{ }^{\circ} \mathrm{C}$.

\section{CONCLUSIONS}

Entanglement density is a key parameter determining solid-state drawability of polyethylene's. Therefore, understanding the effect of melting on entanglement density of low-entangled UHMWPE is of significant importance for production of high-performance fibers by ultra-drawing of solutioncrystallized UHMWPE, as solid-state drawing is performed at elevated temperatures allowing for higher draw ratios to be achieved, leading to improved ultimate properties of the final product. Understanding the effect of melting on entanglement density of low-entangled UHMWPEs is of a significant importance 
since solid-state drawing is performed at elevated temperatures allowing to reach a higher draw ratio which leads to better ultimate properties of drawn materials. ${ }^{10,66,68,69}$ There is a longstanding discussion about the melting-induced change of entanglement density in low-entangled HDPE and UHMWPE, with two contradicting observations. Namely, a rapid "chain explosion" mechanism which causes fast equilibration of entanglement density upon melting, ${ }^{26}$ and meltmemory effect with slow equilibration over time in the molten state. ${ }^{25}$ To gain an insight in the timescale of entanglement formation upon melting of low-entangled UHMWPE, a series of solution-crystallized samples together with a melt-crystallized one were studied. ${ }^{1} \mathrm{H}$ NMR $T_{2}$ relaxometry was used to determine the melting-induced change in the total density of physical junctions that originate from crystal-induced junctions and chain entanglements in the amorphous phase of mc-UH and sc-UH. Since the structure of the entanglement network is affected by reorganization of the semi-crystalline morphology during melting, time-resolved synchrotron WAXS and SAXS experiments were performed during a temperature ramp in the melting temperature range, to follow the changes in crystallinity, crystal thickness and thickness of interlamellar amorphous layers during melting.

The crystallinity of sc-UH is approximately double compared to mc-UH, which is common for low-entangled UHMWPEs. The thickness of interlamellar amorphous layers in sc-UH-1.3, $L_{\mathrm{a}}$, equals $\approx 2 \mathrm{~nm}$, which is approximately the size of chain folds separating adjacent crystal lamellae. The thickness of amorphous layers in sc-UH progressively increases upon melting and finally exceeds $13 \mathrm{~nm}$, i.e. to become close to the initial size of the amorphous layer thickness for the melt-crystallized UHMWPE. This evolution of $L_{\mathrm{a}}$ in sc-UH could facilitate a progressive increase of the entanglement density during melting. Contrary to sc-UH films, the amorphous and crystalline thicknesses in mc-UH did not change much during melting, as the high concentration of chain entanglements in the amorphous phase of mc-UH film are likely to strongly hinder lamellar thickening.

Using ${ }^{1} \mathrm{H}$ NMR $T_{2}$ relaxometry, the total density of physical junctions in the amorphous phase in sc-UH was found to be significantly lower than the one in mc-UH. This is due to the higher fraction of adjacent chain reentering into the same crystal lamella, combined with the lower entanglement density in sc-UH films. Since the contribution of the polymer crystals to the total density of physical junctions vanishes at low crystallinity, chain entanglements provide the major contribution to the network density close to the end of melting. At the initial stage of melting, mc-UH has much higher density of crystal-induced physical junctions than that in partially molten sc-UH (Figure 3). However, the difference gradually disappears with decreasing crystallinity and approaches nearly the same value at the end of melting, suggesting an approximately equal entanglement density at the end of melting. This fast increase in the entanglement density of sc-UH during melting cannot be explained by chain reptation, as the reptation time of the studied UHMWPE exceeds 2 hours. A localized "chain explosion", which occurs in amorphous domains and involves only a fraction of chain coils, is proposed to explain the observed increase in entanglement density. Non-equilibrium super-folded chain configuration with small radius of gyration changes locally in amorphous domains to randomcoil-configuration already during melting and leads to random-coil-configuration with larger $R_{\mathrm{g}}$ at the end of melting. In addition, local entangling occurs at the intermediate stage of melting due to the interweaving of chain segments upon lamellar thickening and their displacements due to volume increase, caused by the melting of crystals.

Thus, the present study shows that a localized "chain explosion" already occurs locally in the amorphous phase during partial melting of solution-crystallized UHMWPE. The solid-state 
drawability behavior of solution-crystallized UHMWPE films, which were exposed to partial melting, confirms this conclusion. The maximum draw ratio decreases and the nominal stress increases upon decreasing crystallinity in melting temperature range, in agreement with past observations. ${ }^{11}$

\section{ASSOCIATED CONTENT}

\section{Supporting Information}

The Supporting Information is available free of charge on the ACS Publications website at DOI.

Results of DSC analysis; ${ }^{1} \mathrm{H}$ NMR $T_{2}$ relaxation experiments; ssynchrotron X-ray scattering analysis.

\section{AUTHOR INFORMATION}

Corresponding Author

Victor Litvinov - V.Lit.Consult, 6191WV Beek, The Netherlands; orcid.org/0000-00015098-9230; Email: v.lit.con@kpnmail.nl

\section{Authors}

Fotis Christakopoulos - ETH Zürich, Vladimir-Prelog-Weg 5, 8093 Zürich, Switzerland; orcid.org/0000-0001-7383-5875

Egor Bersenev - Lomonosov Moscow State University, 119991 Moscow, Russian Federation; Moscow; Institute of Problems of Chemical Physics, Russian Academy of Sciences, Chernogolovka, Moscow Region 142432, Russian Federation

Souren Grigorian - Institute of Physics, University of Siegen, D-57068 Siegen, Germany

André Brem - ETH Zürich, Vladimir-Prelog-Weg 5, 8093 Zürich, Switzerland

Dimitri A. Ivanov - Institut de Sciences des Matériaux de Mulhouse-IS2M, F-68057 Mulhouse, France; Lomonosov Moscow State University, 119991 Moscow, Russian Federation; Moscow; Institute of Problems of Chemical Physics, Russian Academy of Sciences, Chernogolovka, Moscow Region 142432, Russian Federation, orcid.org/0000-0002-5905-2652

Theo Tervoort - ETH Zürich, Vladimir-Prelog-Weg 5, 8093 Zürich, Switzerland; orcid.org/0000-0002-4962-9841

\section{Notes}

The authors declare no competing financial interest.

\section{AKNOWLEDGEMENTS}

V. L. and F. C. thank Prof. Roberto Simonutti and Dr. Michele Mauri from University of Milano Bicocca (Milano, Italy) for hospitality during performing NMR $T_{2}$ relaxation experiment on Minispec MQ20. V. L. thanks Prof. Evelyne van Ruymbeke from Université Catholique de Louvain (Louvain-La-Neuve, Belgium) for providing the equation which is used for estimating the reptation time of the studied UHMWPE. V. L. appreciate financial support from Sabic Technology and Innovation, The Netherlands, and discussion with Rudy Deblieck and Mark Boerakker at an early stage of the project. F.C gratefully acknowledges 
funding by SABIC. D. I. declares that XRD work was performed in accordance with the State Task No AA19-119101590029-0. D. I. appreciates the financial support from Russian Foundation for Basic Research (project no. 19-29-12049). D. I. and E. B. acknowledge the European Synchrotron Radiation Facility (ESRF, Grenoble) for provision of synchrotron beamtime and thank the personnel of the ID02 beamline for perfect technical support. D. I. and E. B. are grateful to the team of the P03 MiNaXS beamline at PETRA III (Hamburg, Germany) and thank them for all their assistance and hospitality during the SAXS measurements session. The authors thank Martin Rosenthal from the BM26 beamline of the ESRF for the help, assistance and useful discussions during the measurements.

\section{REFERENCES}

${ }^{1}$ Iwata, K. Role of entanglements in crystalline polymers 1. Basic theory. Polymer 2002, 43, 6609-6626.

${ }^{2}$ Men, Y.; Rieger, J.; Strobl, G. Role of the entangled amorphous network in tensile deformation of semicrystalline polymers. Phys. Rev. Letters 2003, 91, 095502(R).

${ }^{3}$ Seguela, R. Critical review of the molecular topology of semicrystalline polymers: The origin and assessment of intercrystalline tie molecules and chain entanglements. J. Polym. Sci.: Part B: Polym. Phys. 2005, 43, 1729-1748.

${ }^{4}$ Seguela, R. On the natural draw ratio of semi-crystalline polymers: Review of the mechanical, physical and molecular aspects. Macromol. Mater. Engineering 2007, 292, 235-244.

${ }^{5}$ Deblieck, R.; Van Beek, L.; Remerie, K.; Ward, I. M. Failure mechanisms in polyolefines: The role of crazing, shear yielding and the entanglement network. Polymer 2011, 52, 2979-2990.

${ }^{6}$ Okada, K. N.; Hikosaka, M. Polymer Nucleation. In Handbook of Polymer Crystallization. Piorkowska, E.; Rutledge, G. C., Eds.; John Wiley \& Sons, Inc: Hoboken, New Jersey, 2013.

${ }^{7}$ Wang, S.; Yuan, S.; Wang, K.; Chen, W.; Yamada, K.; Barkley, D.; Koga, T.; Hong, Y.-1.; Miyoshi, T. Intramolecular and intermolecular packing in polymer crystallization. Macromolecules 2019, 52, 4739-4748.

${ }^{8} \mathrm{Hu}, \mathrm{W}$; Schmidt-Rohr, K. Polymer ultradrawability: the crucial role of alpha-relaxation chain mobility in the crystallites. Acta Polym. 1999, 50, 271-285.

${ }^{9}$ Bastiaansen, C. W. M. Influence of initial polymer concentration in solution and weightaverage molecular weight on the drawing behavior of polyethylenes. J. Polym. Sci.: Part B: Polym. Phys. 1990, 28, 1475-1482.

${ }^{10}$ Smith, P.; Lemstra, P. J.; Booij, H. C. Ultradrawing of high- molecular- weight polyethylene cast from solution. II. Influence of initial polymer concentration. J. Polym. Sci.: Polym. Phys. Ed. 1981, 19, 877-888.

11 Bastiaansen, C. W. M.; Meyer, H. E. H.; Lemstra, P. J. Memory effects in polyetylenes: Influence of processing and crystallization history. Polymer 1990, 31, 1435-1440.

12 Kanamoto, T.; Tsuruta, A.; Tanaka, K.; Takeda, M.; Porter, R. Super-drawing of ultrahigh molecular weight polyethylene. 1. Effect of techniques on drawing of single crystal mats. Macromolecules 1988, 21, 470-477.

13 Seoul, C.; Kim, S. Y. Single- crystal- like orientation of ultrahigh- molecular- weight polyethylene by uniaxial stretching. J. Polym. Sci.: Part B: Polym. Phys. 1988, 26, 19651978. 
${ }^{14}$ Flory, P. J.; Yoon, D. Y. Molecular morphology in semicrystalline polymers. Nature 1978, 272, 226-229.

${ }^{15}$ Chuanfu, L.; Sommer, J.-U. Disentanglement of linear polymer chains toward unentangled crystals. ACS Macro Lett. 2013, 2, 31-34.

${ }^{16}$ Kurz, R.; Schulz, M.; Scheliga, A.; Men, Y.; Seidlitz, A.; Thurn-Albrecht, T.; Saalwächter, K. Interplay between crystallization and entanglements in the amorphous phase of the crystalfixed polymer poly(e-caprolactone). Macromolecules 2018, 51, 5831-5841.

17 Strobl, G. The Physics of Polymers. Concepts for Understanding Their Structures and Behavior; Springer: New York, 1997.

${ }^{18}$ Lotz, B.; Miyoshi, T.; Cheng, S. Z. D. $50^{\text {th }}$ Anniversary perspective: Polymer crystals and crystallization: Personal journeys in a challenging research field. Macromolecules 2017, 50, 5995-6025.

${ }^{19}$ Archer, L. A. Polymer disentanglement in steady-shear flow. Rheologica Acta 2001, 40, 7485 .

${ }^{20}$ Luo, C.; Kröger, M.; Sommer, J.-U. Entanglements and crystallization of concentrated polymer solutions: Molecular dynamics simulations. Macromolecules 2016, 49, 9017-9025.

${ }^{21}$ Brem, A.; Lhost, O.; Tervoort, T. A. Influence of solvent quality and crystallization conditions on the drawability of ultra-high molecular weight polyethylene cast from solution. Macromolecules 2020, 53, 5957-5970.

${ }^{22}$ Rotzinger B.; Chanzy H.; Smith P. High strength/high modulus polyethylene: synthesis and processing of ultra-high molecular weight virgin powders. Polymer 1989, 30, 1814-1819.

${ }^{23}$ Romano, D.; Andablo-Reyes, E.; Ronca, S.; Rastogi, S. Aluminoxane co-catalysts for the activation of a bis phenoxyimine titanium (IV) catalyst in the synthesis of disentangled ultrahigh molecular weight polyethylene. Polymer 2015, 74, 76-85.

${ }^{24}$ Bassett, D. C. Chain-extended polyethylene in context: a review. Polymer 1976, 17, 460-470.

${ }^{25}$ Michell, R. M.; Mugica, A.; Zubitur, M.; Müller, A. J. Self-nucleation of crystalline phases within homopolymers, polymer blends, copolymers, and nanocomposites. Adv. Polym. Sci. 2015, 327, 1-42; and refs. therein.

${ }^{26}$ Barham, P. J.; Sadler, D. M. A neutron scattering study of the melting behavior of polyethylene single crystals. Polymer 1991, 32, 393-395.

${ }^{27}$ de Gennes, P. G. Explosion à la fusion. C. R. Acad. Sci., Sér. IIb: Méc., Phys., Chim., Astron. 1995, 321, 363-365.

${ }^{28}$ Psarski, M.; Piorkowska, E.; Galeski, A. Crystallization of polyethylene from melt with lowered chain entanglements. Macromolecules 2000, 33, 916-932.

${ }^{29}$ Rastogi, S.; Lippits, D. R.; Peters, G. W. M.; Graf, R.; Yao, Y.; Spiess, H. W. Heterogeneity in polymer melts from melting of polymer crystals. Nature Materials 2005, 4, 835-841.

30 Lippits, D. R.; Rastogi, S.; Höhne, G. W. H.; Mezari. B.; Magusin, P. C. M. M. Heterogeneous distribution of entanglements in the polymer melt and its influence on crystallization. Macromolecules 2007, 40, 1004-1010.

31 Yao, Y.; Jiang, S.; Rastogi, S. ${ }^{13} \mathrm{C}$ solid state NMR characterization of structure and orientation development in the narrow and broad molar mass disentangled UHMWPE. Macromolecules 2014, 47, 1311-1382.

${ }^{32}$ Xue, Y.-Q.; Tervoort, T. A.; Lemstra, P. J. Welding behavior of semicrystalline polymers. 1. The effect of nonequilibrium chain conformations on autoadhesion of UHMWPE. Macromolecules 1998, 31, 3075-3080. 
${ }^{33}$ Xue, Y.-Q.; Tervoort, T. A.; Rastogi, S.; Lemstra, P. J. Welding behavior of semicrystalline polymers. 2. Effect of cocrystallization on autoadhesion. Macromolecules 2000, 33, 70847087.

${ }^{34}$ Deplanke, T.; Lame, O.; Rousset, F.; Aguili, I.; Seguela, R.; Vigier, G. Diffusion versus cocrystallization of very long polymer chains at interfaces: Experimental study of sintering of UHMWPE nascent powder. Macromolecules 2014, 47, 197-207.

${ }^{35}$ Litvinov, V.; Deblieck, R.; Clair, C.; Van den fonteyne, W.; Lallam, A.; Kleppinger, R.; Ivanov, D. A.; Ries, M. E.; Boerakker, M. Molecular structure, phase composition, melting behavior and chain entanglements in the amorphous phase of high-density polyethylenes. Macromolecules 2020, 53, 5418-54133.

${ }^{36}$ Hocquet, S.; Dosière, M.; Thierry, A.; Lotz, B.; Koch, M. H. J.; Dubreuil, N.; Ivanov, D. A. Morphology and melting of truncated single crystals of linear polyethylene. Macromolecules 2003, 36, 8376-8384.

${ }^{37}$ Magonov, S. N.; Yerina, N. A.; Ungar, G.; Reneker, D. H.; Ivanov, D. A. Chain unfolding in single crystals of ultra-long alkane $\mathrm{C}_{390} \mathrm{H}_{782}$ and polyethylene: an atomic force microscopy study. Macromolecules 2003, 36, 5637-5649.

${ }^{38}$ Dubreuil, N.; Hocquet, S.; Dosière, M.; Ivanov, D. A. Melting of isochronously decorated single crystal of linear polyethylene, as monitored with atomic force microscopy. Macromolecules 2004, 37, 1-5.

${ }^{39}$ Wunderlich, B.; Cormier, C. M. Heat of fusion of polyethylene. J. Polym. Sci.: Part A-2: Polym. Phys. 1967, 5, 987-988.

${ }^{40}$ Sztucki, M.; Narayanan, T. Development of an ultra-small-angle X-ray scattering instrument for probing the microstructure and the dynamics of soft matter. J. Appl. Crystallogr. 2007, 40, 459-462.

${ }^{41}$ Ashiotis, G.; Deschildre, A.; Nawaz, Z.; Wright, J. P.; Karkoulis, D.; Picca, F. E.; Kieffer, J. The fast azimuthal integration Python library: pyFAI. J. Appl. Cryst. 2015, 48, 510-519.

${ }^{42}$ Strobl, G. R.; Schneider, M. J.; Voigt-Martin, I. G. Model of partial crystallization and melting derived from small- angle $\mathrm{X}$ - ray scattering and electron microscopic studies on low- density polyethylene. J. Polym. Sci. Polym. Phys. Ed. 1980, 18, 1361-1381.

${ }^{43}$ Ruland, W. The evaluation of the small-angle scattering of lamellar two-phase systems by means of interface distribution functions. Colloid Polym. Sci. 1977, 255, 417-427.

${ }^{44}$ Ivanov, D. A.; Hocquet, S.; Dosière, M.; Koch, M. Exploring the melting of a semirigid chain polymer with synchrotron time- and temperature-resolved small-angle X-ray scattering. Eur. Phys. J. E 2004, 13, 363-378.

${ }^{45}$ Ivanov, D. A. ; Bar, G. ; Dosière, M. ; Koch, M. H. J. A novel view on crystallization and melting of semirigid chain polymers: The case of poly(trimethylene terephthalate). Macromolecules 2008,41, 9224-9231.

${ }^{46}$ Litvinov, V. M.; van Duin, M. Real-time ${ }^{1} \mathrm{H}$ NMR relaxation study of EPDM vulcanization. Kautsch. Gummi Kunstst. 2002, 55, 460-463.

${ }^{47}$ Agarwal, V.; van Erp, T. B.; Balzano, L.; Gahleitner, M.; Parkinson, M.; Govaert, L. E.; Litvinov, V.; Kentgens, A. P. M. The chemical structure of the amorphous phase of propylene-ethylene random copolymers in relation to their stress-strain properties. Polymer 2014, 55, 896-905. 
${ }^{48}$ Qin, Y.; Qiao, Y.; Chassé, W.; Litvinov, V.; Men, Y. Crystallinity of polyolefins with large side groups by low-field ${ }^{1} \mathrm{H}$ NMR $T_{2}$ relaxometry: Isotactic polybutene-1 with form II and crystals. Solid State Nuclear Magn. Res. 2020, 105, 101637

49 Litvinov, V. M. Characterization of chemical and physical networks in rubbery materials using proton NMR magnetization relaxation. In: Spectroscopy of Rubbers and Rubbery Materials. Litvinov, V. M.; De, P. P., Eds.; Rapra Technology: Shawbury, 2002.

${ }^{50}$ Saalwächter, K. Proton multiple-quantum NMR for the study of chain dynamics and structural constraints in polymeric soft materials. Prog. Nucl. Magn. Res. Spectr. 2007, 51, 1-35.

51 Höpfner, J.; Guthausen, G.; Saalwächter, K.; Wilhelm, M. Network structure and inhomogeneities of model and commercial polyelectrolyte hydrogels as investigated by lowfield proton NMR techniques. Macromolecules 2014, 47, 4251-4265.

52 Litvinov, V. M.; Barendswaard, W.; van Duin, M. The density of chemical crosslinks and chain entanglements in unfilled EPDM vulcanizates as studied with low resolution, solid state ${ }^{1}$ H NMR. Rubber Chemistry Technology 1998, 71, 105-118.

${ }^{53}$ Litvinov, V. M.; Braam, A. W. M.; van der Ploeg, A. F. M. J. Telechelic ionomers: Molecular structure and kinetics of physical gelation of unsaturated polyester as studied by solid state NMR and X-ray. Macromolecules 2001, 34, 489-502.

${ }^{54}$ Litvinov, V. M. EPDM/PP thermoplastic vulcanizates as studied by proton NMR relaxation: Phase composition, molecular mobility, network structure in the rubbery phase and network heterogeneity. Macromolecules 2006, 39, 8727-8741.

55 Litvinov, V. M.; Ries, M. E.; Baughman, T. W.; Henke, A.; Matloka, P. P. Chain entanglements in polyethylene melts. Why it is studied again? Macromolecules 2013, 46, 541-547.

${ }^{56}$ Hall, K. W.; Sirk, T. W.; Klein, M. L.; Shinoda, W. A coarse-grain model for entangled polyethylene melts and polyethylene crystallization. J. Chem. Phys. 2019, 150, 244901; and refs. therein.

57 Teng, C.; Gao, Y.; Wang, X.; Jiang, W.; Zhang, C.; Wang, R.; Zhou, D.; Xue, G. Reentanglement kinetics of freeze-dried polymers above the glass transition temperature. Macromolecules 2012, 45, 6648-6651.

${ }^{58}$ Uehera, H.; Yamanobe, T.; Komoto, T. Relationship between solid-state molecular motion and morphology for ultra-high molecular weight polyethylene crystallized under different conditions. Macromolecules 2000, 33, 4861-4870.

${ }^{59}$ Yao, Y.- F.; Graf, R.; Spiess, H. W.; Lippits, D. R.; Rastogi, S. Morphological differences in semicrystalline polymers: Implications for local dynamics and chain diffusion. Phys. Rev. E 2007, 76, 060801(R).

${ }^{60}$ Litvinov, V. Molecular Mobility and Phase Composition in Polyolefines: From Fundamental to Applied Research, in NMR Spectroscopy of Polymers: Innovative Strategies for Complex Macromolecules, ACS Symposium Series; Cheng, H. N.; Asakura, T.; English, A. D., Eds.; 2011; Vol. 1077, Chapter 11, pp 179-190.

${ }^{61}$ Fatkullin, N.; Fischer, E.; Mattea, C.; Beginn, U.; Kimmich, R. Polymer dynamics under nanoscopic constraints: The "corset effect" as revealed by NMR relaxometry and diffusometry. ChemPhysChem 2004, 5, 884-894.

${ }^{62}$ Yamazaki, S.; Gu, F.; Watanabe, K; Okada, K.; Toda, A.; Hikosaka, M. Two-step formation of entanglement from disentangled polymer melt detected by using nucleation rate. Polymer 2006, 47, 6422-6428. 
${ }^{63}$ Allegra, G.; Famulari, A. Chain statistics in polyethylene crystallization. Polymer 2009, 50, $1819-1829$.

${ }^{64}$ Matsuda, H.; Aoike, T.; Uehara, H.; Yamanobe, T.; Komoto, T. Overlapping of different rearrangement mechanisms upon annealing for solution-crystallized polyethylene. Polymer 2001, 42, 5013-5021.

${ }^{65}$ Ottani, S.; Ferracini, E.; Ferrero, A.; Malta, V.; Porter, R. S. A latent entanglement model: Effects of irregularities on the crystalline surface on polymer draw. Macromolecules 1996, 29, $5326-5331$.

${ }^{66}$ Lemstra, P. J.; Bastiaansen, C. W. M.; Peij, T,; Jacobs, M. J. N. Fibres Based on Ultra-High Molecular Weight Polyethylene - Processing and Applications. In Solid Phase Processing of Polymers; Ward, I. M.; Coates, P. D.; Dumoulin, M. M., Eds; Hanser Publishers: Munich, 2000, pp 172-213.

${ }^{67}$ Colby, R. H.; Fetters, L. J.; Funk, W. G.; Graessley, W. W. Effects of concentration and thermodynamic interaction on the viscoelastic properties of polymer solutions. Macromolecules 1991, 24. 3873-82.

${ }^{68}$ Capaccio, G.; Crompton, T. A.; Ward, I. M. Drawing behavior of linear polyethylene. II. Effect of draw temperature and molecular weight on draw ratio and modulus. J. Polym. Sci.:Polymer. Phys. Ed. 1980, 18, 301-309.

${ }^{69}$ Barham, P. G.; Keller, A. Review. High-strength polyethylene fibres from solution and gel spinning. J. Mater. Sci. 1985, 20, 2281-2302. 